where

$$
f(x)=r_{0}+r_{1} x+\ldots+r_{p-2} x^{p-2},
$$

$Z=e^{2 i \pi /(p-1)}$, and $r_{i}$ is the least positive residue of $r^{i}$, modulo $p$. Mr. Vandiver derives necessary and sufficient conditions that $h$ be divisible by $p^{n}$, in terms of Bernoulli numbers, the argument used being different from those employed by Kummer and Kronecker in their treatment of the case $n=1$.

20. In his first proof of the law of reciprocity between two ideals in a regular cyclotomic field Kummer gave the relation*

where

$$
\left[\frac{d^{p-1} \log \omega\left(e^{v}\right)}{d v^{p-1}}\right]_{v=0} \equiv \frac{(\omega(1))^{p-1}-N(\omega)}{p}(\bmod p),
$$

$$
\begin{aligned}
\omega(x) & =a_{0}+a_{1} x+\ldots+a_{p-2} x^{p-2}, \\
\omega & =a_{0}+a_{1} \alpha+\ldots+a_{p-2} \alpha^{p-2},
\end{aligned}
$$

$\alpha=e^{2 i \pi / p}$ and $a_{0}, a_{1}, \ldots, a_{p-2}$ are integers, $\omega$ being prime to the prime $p$. In the present paper Mr. Vandiver gives a proof of this result which differs in character from that of Kummer.

F. N. Cole, Secretary.

\title{
NOTE ON ISOGENOUS COMPLEX FUNCTIONS OF CURVES.
}

BY PROFESSOR W. C. GRAUSTEIN.

(Read before the American Mathematical Society September 5, 1917.)

LET $L$ be a continuous, closed and directed space curve, without multiple points, let $-L$ be the same curve oppositely directed, and let $F|[L]|$ be a function of $L$, such that $F|[-L]|=-F|[L]|$. Form the ratio

$$
\frac{F\left|\left[L^{\prime}\right]\right|-F|[L]|}{\sigma},
$$

where $L^{\prime}$ is the directed curve obtained from $L$ by replacing an arc $P P^{\prime}$ of $L$ by a second arc joining $P$ with $P^{\prime}$, and $\sigma$

* Abhandlungen, Berlin Academy, 1859, p. 119, formula (7). 
is the area of a surface $S$ bounded by the two arcs $P P^{\prime}$. The limit of this ratio as the surface $S$ shrinks to a point in $P$, if it exists, is called the derivative of $F|[L]|$ with respect to $S$ in the point $P$ and is denoted by $d F / d \sigma$. This derivative, dependent in general on $L$ as well as on $P$ and $S$, becomes for a given $S$ a function of $P$ alone, in the case that $F|[L]|$ is a so-called function of the first degree, that is, is a function such that the sum of its values for two directed curves, possessing a common arc oppositely directed for the two, is equal to its value for the curve resulting from the combination of the parts of the two curves other than their common arc. In this case the above ratio becomes $F\left|\left[L^{\prime \prime}\right]\right| / \sigma$, where $L^{\prime \prime}$ is the directed boundary of $S$, made up of the directed arcs $P P^{\prime}$ of $L^{\prime}$ and $-L$. Furthermore, in this case,

$$
F\left|\left[L^{\prime \prime}\right]\right|=\int_{S} \int(p \cos n x+q \cos n y+r \cos n z) d \sigma,
$$

where $p, q, r$ are continuous point functions and $\cos n x$, $\cos n y, \cos n z$ are the direction cosines of the normal to $S$ suitably directed. Then the value of the derivative of $F$ with respect to $S$ in the point $P$ is

$$
\frac{d F}{d \sigma}=\lim _{\sigma=0} \frac{F\left|\left[L^{\prime \prime}\right]\right|}{\sigma}=p \cos n x+q \cos n y+r \cos n z,
$$

provided that the values of $p, q, r, \cos n x, \cos n y, \cos n z$ are taken in $P . *$

The functions $p, q, r$ are termed the derivatives of $F$ in $P$ with respect to the three coordinate planes. Consider them as the components of a vector point function or vector $c$, and call this vector $c$ the gradient of the function $F$ in $P$. If the gradient $c$, besides being continuous, possesses continuous first partial derivatives, it follows, by application of the theorem of Gauss expressing a volume integral in terms of a surface integral, that

$$
\operatorname{div} c=\frac{\partial p}{\partial x}+\frac{\partial q}{\partial y}+\frac{\partial r}{\partial z}=0 .
$$

* For a more detailed development of the contents of this and the two following paragraphs, see Volterra, "Sur une généralisation de la théorie des fonctions d'une variable imaginaire," Acta Mathematica, vol. 12 (1889), pp. 233-286; Lévy, "Sur les equations intégro-differentielles définissant des fonctions de lignes," Doctor's Thesis, Paris, 1911, Introduction; Evans, "Topics from the theory and applications of functionals, including integral equations," The Cambridge Colloquium Lectures, delivered September 6-8, 1916, Lectures I, II. 
Conversely, if $\operatorname{div} c=0$, where $c$ is a vector continuous with continuous first partial derivatives, there exists a function of curves, $F|[L]|$, of which $c$ is the gradient.

Consider now

where

$$
F=F_{1}+i F_{2}, \quad \Phi=\Phi_{1}+i \Phi_{2},
$$

$F_{1}=F_{1}|[L]|, \quad F_{2}=F_{2}|[L]|, \quad \Phi_{1}=\Phi_{1}|[L]|, \quad \Phi_{2}=\Phi_{2}|[L]|$

are functions of $L$ of the first degree possessing gradients $c_{1}, c_{2}, \gamma_{1}, \gamma_{2}$, which are continuous with continuous first partial derivatives. Form the ratio $\Delta \Phi / \Delta F$, where $\Delta F$ and $\Delta \Phi$ are the variations in $F$ and $\Phi$ due to replacing the $\operatorname{arc} P P^{\prime}$ of $L$ by a second arc joining $P$ with $P^{\prime}$. Evidently the limit, $d \Phi / d F$, of this ratio, as the surface $S$ bounded by the two arcs $P P^{\prime}$ shrinks to a point in $P$, is $(d \Phi / d \sigma):(d F / d \sigma)$. It depends, in general, not merely on $P$ but on the aspect of the surface $S$ in $P$, namely, on the values of $\cos n x, \cos n y, \cos n z$ in $P$. The condition necessary and sufficient that it be independent of $S$ is that the vectors $c_{1}+i c_{2}, \gamma_{1}+i \gamma_{2}$ be proportional, or, in other words, that the vector product $\overline{c_{1}+i c_{2}} \times \overline{\gamma_{1}+i \gamma_{2}}$ vanish, that is, that

$$
c_{1} \times \gamma_{1}-c_{2} \times \gamma_{2}=0, \quad c_{1} \times \gamma_{2}+c_{2} \times \gamma_{1}=0 .
$$

If these conditions (1) are satisfied, $d \Phi / d F$ is dependent only on $P$, and the two complex functions $F=F_{1}+i F_{2}, \Phi=\Phi_{1}+i \Phi_{2}$ are said to be isogenous.

Lévy has noted,* without going into the details of hypothesis or proof, the general fact that for a given complex function $F=F_{1}+i F_{2}$ there exist complex functions $\Phi=\Phi_{1}+i \Phi_{2}$ isogenous to it, depending on two arbitrary functions of two independent parameters. In proving this, we assume that the functions $F_{1}, F_{2}$ possess gradients $c_{1}, c_{2}$, which are analytic, in general, in the coordinates $x, y, z$ of the point $P$. If, then, we can establish the existence of analytic vectors $\gamma_{1}, \gamma_{2}$, satisfying the equations (1) and having divergences equal to zero, the existence of functions $\Phi=\Phi_{1}+i \Phi_{2}$ isogenous to $F=F_{1}+i F_{2}$ will follow.

The vectors $\gamma_{1}, \gamma_{2}$ will satisfy (1) when and only when they are linear combinations of the vectors $c_{1}, c_{2}$ of the form

* Loc. cit., p. 11. 


$$
\gamma_{1}=k_{1} c_{1}+k_{2} c_{2}, \quad \gamma_{2}=k_{2} c_{1}-k_{1} c_{2},
$$

where $k_{1}$ and $k_{2}$ are scalar point functions. Thus our problem reduces to the determination of $k_{1}, k_{2}$ as solutions of the equations

$$
\operatorname{div}\left(k_{1} c_{1}+k_{2} c_{2}\right)=0, \quad \operatorname{div}\left(k_{2} c_{1}-k_{1} c_{2}\right)=0,
$$

or better, as solutions of the equations (2), to which these reduce by virtue of the vanishing of the divergences of $c_{1}, c_{2}$,

$$
\begin{aligned}
& p_{1} \frac{\partial k_{1}}{\partial x}+q_{1} \frac{\partial k_{1}}{\partial y}+r_{1} \frac{\partial k_{1}}{\partial z}+p_{2} \frac{\partial k_{2}}{\partial x}+q_{2} \frac{\partial k_{2}}{\partial y}+r_{2} \frac{\partial k_{2}}{\partial z}=0, \\
& p_{2} \frac{\partial k_{1}}{\partial x}+q_{2} \frac{\partial k_{1}}{\partial y}+r_{2} \frac{\partial k_{1}}{\partial z}-p_{1} \frac{\partial k_{2}}{\partial x}-q_{1} \frac{\partial k_{2}}{\partial y}-r_{1} \frac{\partial k_{2}}{\partial z}=0 .
\end{aligned}
$$

But according to the theory of partial differential equations there exist simultaneous solutions $k_{1}, k_{2}$ of the equations (2) which are analytic in general and depend on two arbitrary functions of two independent variables.

To go more into detail concerning these solutions, let us consider instead of the equations (2) the equivalent equations (3), obtained by solving the equations (2) for $\partial k_{1} / \partial z, \partial k_{2} / \partial z$,

$$
\begin{aligned}
\left(r_{1}^{2}+r_{2}^{2}\right) \frac{\partial k_{1}}{\partial z}+ & \left(p_{1} r_{1}+p_{2} r_{2}\right) \frac{\partial k_{1}}{\partial x}+\left(q_{1} r_{1}+q_{2} r_{2}\right) \frac{\partial k_{1}}{\partial y} \\
& +\left(p_{2} r_{1}-p_{1} r_{2}\right) \frac{\partial k_{2}}{\partial x}+\left(q_{2} r_{1}-q_{1} r_{2}\right) \frac{\partial k_{2}}{\partial y}=0, \\
\left(r_{1}^{2}+r_{2}^{2}\right) \frac{\partial k_{2}}{\partial z}+ & \left(p_{1} r_{2}-p_{2} r_{1}\right) \frac{\partial k_{1}}{\partial x}+\left(q_{1} r_{2}-q_{2} r_{1}\right) \frac{\partial k_{1}}{\partial y} \\
& +\left(p_{1} r_{1}+p_{2} r_{2}\right) \frac{\partial k_{2}}{\partial x}+\left(q_{1} r_{1}+q_{2} r_{2}\right) \frac{\partial k_{2}}{\partial y}=0 .
\end{aligned}
$$

There exist unique solutions $k_{1}, k_{2}$ of the equations (3), analytic in the neighborhood of $\left(x_{0}, y_{0}, z_{0}\right)$, taking for $z=z_{0}$ given values $m_{1}(x, y), m_{2}(x, y)$, and possessing in $\left(x_{0}, y_{0}, z_{0}\right)$ given partial derivatives $\left(\partial k_{1} / \partial x\right)_{0},\left(\partial k_{1} / \partial y\right)_{0},\left(\partial k_{2} / \partial x\right)_{0},\left(\partial k_{2} / \partial y\right)_{0}$, provided that the functions to which $\partial k_{1} / \partial z$ and $\partial k_{2} / \partial z$ are actually equal in equations (3) are themselves analytic in the neighborhood of $\left(x_{0}, y_{0}, z_{0},\left(\partial k_{1} / \partial x\right)_{0},\left(\partial k_{1} / \partial y\right)_{0},\left(\partial k_{2} / \partial x\right)_{0}\right.$, $\left.\left(\partial k_{2} / \partial y\right)_{0}\right), *$ that is to say, provided that the vectors $c_{1}, c_{2}$ are

* Cf., e. g., Goursat, Cours d'Analyse mathématique, vol. 2, p. 363. 
analytic in $\left(x_{0}, y_{0}, z_{0}\right)$ and $r_{1}$ and $r_{2}$ are not both zero there. These solutions of (3) are, then, analytic except perhaps in points of singularity of $c_{1}, c_{2}$ and in points for which $r_{1}=r_{2}=0$. But they are identical with certain solutions of the equations (2), solved for $\partial k_{1} / \partial y, \partial k_{2} / \partial y$, - solutions which are analytic except perhaps in points of singularity of $c_{1}, c_{2}$ and in points for which $q_{1}=q_{2}=0$. Evidently, then, these solutions $k_{1}, k_{2}$, and hence the vectors $\gamma_{1}=k_{1} c_{1}+k_{2} c_{2}, \gamma_{2}=k_{2} c_{1}-k_{1} c_{2}$, are analytic except perhaps in points of singularity of $c_{1}, c_{2}$ and in points in which both $c_{1}$ and $c_{2}$ are indeterminate, that is, have all three components zero. Thus we have the theorem:

If the gradients $c_{1}, c_{2}$ of the functions $F_{1}, F_{2}$ in $F=F_{1}+i F_{2}$, where $F_{1}=F_{1}|[L]|, F_{2}=F_{2}|[L]|$ are functions of the first degree of the space curve $L$, are in general analytic, the gradients $\gamma_{1}, \gamma_{2}$ of $\Phi_{1}, \Phi_{2}$ in $\Phi=\Phi_{1}+i \Phi_{2}$, an arbitrary complex function of $L$ of the first degree isogenous to $F$, are analytic save perhaps in points of singularity of $c_{1}$ or $c_{2}$ and points in which both these vectors are indeterminate.

The theorem still holds when the vectors $c_{1}, c_{2}$ are proportional. In this case $k_{1}$ and $k_{2}$ are both solutions of the equation

$$
p_{i} \frac{\partial k}{\partial x}+q_{i} \frac{\partial k}{\partial y}+r_{i} \frac{\partial k}{\partial z}=0, \quad(i=1,2),
$$

to which both of the equations (3) reduce in form, and $\gamma_{1}$ and $\gamma_{2}$ are both proportional to $c_{1}$ and $c_{2}$.

The Rice Institute,

Houston, Texas.

\section{AN ELEMENTARY DERIVATION OF THE PROBABILITY FUNCTION.}

BY CAPTAIN ALBERT A. BENNETT, C.A.R.C.

WE shall derive by means of elementary considerations the equation of the probability curve from the sequence of binomial coefficients. If the asymptotic form of $x !$ be obtained, the problem is very simple but none the less merits attention. The asymptotic form of $n$ !, viz., $\sqrt{2 \pi n}(n / e)^{n} e^{\theta /(12 n)}, 0<\theta<1$, might of course be taken for granted, but so far as is known 